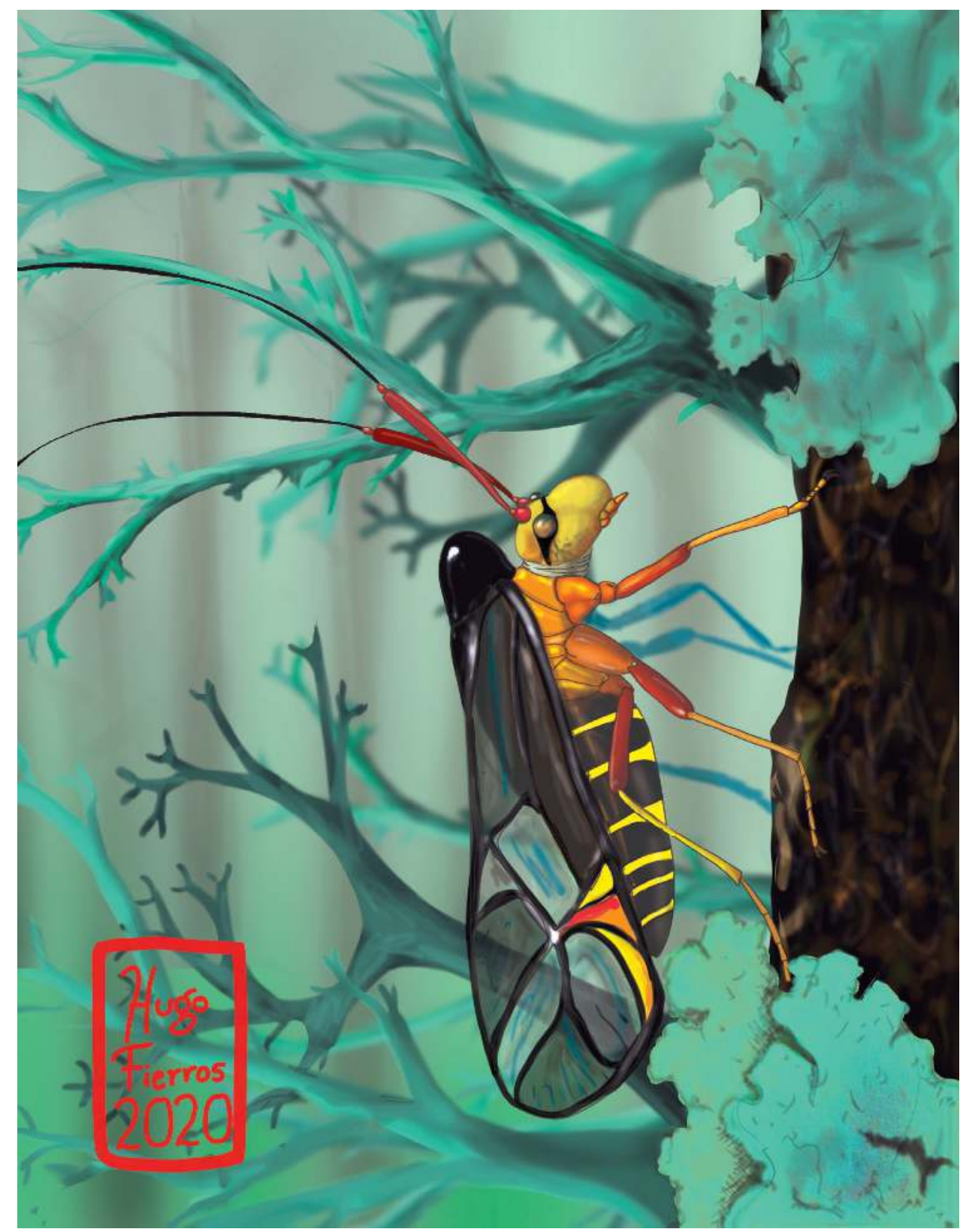

Dugesiana, Año 27, No. 2, julio 2020-diciembre 2020 segundo semestre de 2020), es una publicación semestral, editada por la Universidad de Guadalajara, a través del Centro de Estudios en Zoología, por el Centro Universitario de Ciencias Biológicas y Agropecuarias. Camino Ramón Padilla Sánchez \# 2100, Nextipac, Zapopan, Jalisco, Tel. 37771150 ext. 33218, http://148.202.248.171/dugesiana/index.php/DUG/index, glenusmx@gmail.com. Editor responsable: José Luis Navarrete-Heredia. Reserva de Derechos al Uso Exclusivo 04-2009-062310115100203, ISSN: 2007-9133, otorgados por el Instituto Nacional del Derecho de Autor. Responsable de la última actualización de este número: José Luis Navarrete-Heredia, Editor y Ana Laura González-Hernández, Asistente Editorial. Fecha de la última modificación 1 de julio 2020, con un tiraje de un ejemplar.

Las opiniones expresadas por los autores no necesariamente reflejan la postura del editor de la publicación.

Queda estrictamente prohibida la reproducción total o parcial de los contenidos e imágenes de la publicación sin previa autorización de la Universidad de Guadalajara. 
Artículo

\title{
Hormigas (Hymenoptera: Formicidae) asociadas a dos especies de Psittacanthus (Santalales: Loranthaceae) en Felipe Carrillo Puerto, Quintana Roo, México
}

\author{
Ants (Hymenoptera: Formicidae) associated with two species (Santalales: Loranthaceae) in Felipe Ca- \\ rrillo Puerto, Quintana Roo, Mexico \\ León Esteban Ibarra-Garibay y Gabriela Castaño-Meneses* \\ Laboratorio de Ecología de Artrópodos en Ambientes Extremos, UMDI-Facultad de Ciencias, Universidad Nacio- \\ nal Autónoma de México, Campus Juriquilla, Boulevard Juriquilla 3001, C.P. 76230, Querétaro, México. leoniba- \\ rragar@hotmail.com, "gabycast99@hotmail.com
}

\section{RESUMEN}

En México se tienen pocos estudios sobre artrópodos asociados a plantas parásitas como son los muérdagos, que, desafortunadamente, afectan a gran parte de la masa forestal. Con respecto a las hormigas, sólo se tienen registros de algunas especies asociadas a los nectarios extraflorales de estas plantas, así como de hormigas cortadoras que llegan a consumir sus hojas. En el presente trabajo se estudiaron las hormigas arborícolas asociadas a plantas hemiparásitas del género Psittacanthus en el estado de Quintana Roo. La colecta de hormigas se realizó durante tres podas llevadas a cabo por los integrantes de las Brigadas Comunitarias de Sanidad Forestal del Estado de Quintana Roo, a los árboles atacados por plantas hemiparásitas (Psittacanthus mayanus Standl. \& Steyerm., 1944 y P. rhynchanthus (Benth.) Kuijt, 1987), las ramas afectadas se encontraban a alturas entre 7 y 18 m, en una selva mediana subpereniifolia en el ejido de Felipe Carrillo Puerto (1940'24.8” 8758'34.3”) durante el año 2017. Se encontraron las especies Dolichoderus bispinosus (Olivier, 1792), D. diversus Emery, 1894, Pseudomyrmex kuenckeli (Emery, 1890) y P. simplex (Smith, 1877) en Psittacanthus mayanus infectando a Lysiloma latisiliquum (L.) Benth., 1875, mientras que Camponotus sericeiventris (Guérin-Méneville, 1838), Cephalotes biguttatus (Emery, 1890) y Dolichoderus diversus Emery, 1894, fueron colectados en Psittacanthus rhyntacanthus. Camponotus se encontró en plantas hemiparásitas de Coccoloba spicata Lundell, 1939, mientras que Cephalotes y Dolichoderus en plantas parasitando a Lonchocarpus rugosus Benth., 1860. Son los primeros registros de hormigas asociadas a plantas de la familia Loranthaceae, y se establece el potencial que tienen estas plantas para albergar una biodiversidad considerable. Es importante realizar esfuerzos para conocer el papel que pueden tener las hormigas en estas plantas y las interacciones que establecen con las mismas, debido a que la presencia de plantas parásitas es considerado un problema a nivel nacional, por lo que, esta información puede permitir tener más herramientas que puedan ayudar a desarrollar mejores planes de manejo.

Palabras clave: Artrópodos, Dosel, Interacciones, Muérdago, Plantas parásitas.

\begin{abstract}
There are few studies about arthropods associated to parasitic plants as mistletoes that, unfortunately, affect a great surface of forest mass in our country. In relation to the ants, only some species are recorded associated to extrafloral nectaries of those plants, also cutting ants that can use their leaves. Present work was focused in the arboriculous ants associated to hemiparasitic plants of Psittacanthus genus from Quintana Roo state. Sampling of ants was carried out during pruning campaign carried out by member of the Community Forestry Health Brigades of the State of Quintana Roo, in trees attacked by hemiparasite plants (Psittacanthus mayanus Standl. \& Steyerm., 1944 and $P$. rhynchanthus (Benth.) Kuijt, 1987), the affected branches were found at 7 and 18 meters heights, in a medium subrefinifolious forest, in the ejido Felipe Carrillo Puerto (1940'24.8” 8758'34.3”) during 2017. The ant species Dolichoderus bispinosus (Olivier, 1792), D. diversus Emery, 1894, Pseudomyrmex kuenckeli (Emery, 1890) and P. simplex (Smith, 1877) were found in Psittacanthus mayanus infecting Lysiloma latisiliquum (L.) Benth., 1875 trees, while ants Camponotus sericeiventris (Guérin-Méneville, 1838), Cephalotes biguttatus (Emery, 1890) and Dolichoderus diversus Emery, 1894 were collected in Psittacanthus rhyntacanthus. Camponotus was found in hemiparasitic plants affecting Coccoloba spicata Lundell, 1939, while Cephalotes and Dolichoderus were found in hemiparasitic plants growing on Lonchocarpus rugosus Benth., 1860. There are the first records of those ants associated with Loranthaceae family and the potential of these plants to maintaining a considered biodiversity is established. Efforts directed to know the role of ants and the relationships that they establish in these plants are important, due the presence of parasitic plants is considered a national problem, in order to have useful tools than can help development better management plans.
\end{abstract}

Key words: Artrhopods, Canopy, Interactions, Mistletoe, Parasitic plants.

Las plantas parásitas y hemiparásitas conforman cerca del 1\% de las especies de angiospermas (Press et al. 1999), y son comunes tanto en ambientes naturales como modificados, teniendo efecto importante en sus árboles hospederos (Press y Phoenix 2005). En México, son un importante agente biológico de perturbación, ya que causan graves pér- didas económicas en el ámbito forestal, además de ocasionar la muerte del arbolado y aumentar el riesgo de ataque de plagas y otras enfermedades forestales (Geils et al. 2002; CONAFOR 2006).

Actualmente las plantas parásitas representan un problema a nivel nacional, dado que afectan gran superficie 
de las masas forestales del país, sin embargo, no se han realizado estudios que reflejen la verdadera importancia de estas plantas (Alvarado y Saavedra 2017). Las semillas son dispersadas por aves (Tun-Garrido 2010), al germinar producen una raíz modificada llamada haustorio que penetra en el cuerpo de la planta hospedera, y es por medio de esta raíz que extraen de su hospedero los recursos suficientes (carbohidratos, agua y sales minerales) para completar su ciclo de vida (Alvarado y Saavedra 2017). De la familia Loranthaceae, en México, se conocen cinco géneros y más de 50 especies (Martínez-Ambriz 2017), mientras que para la península de Yucatán sólo se tienen registradas 10 especies incluidas en cuatro géneros (Villaseñor 2016).

Existe poca información sobre artrópodos asociados a plantas parásitas a nivel mundial, si bien en los últimos años se ha puesto más énfasis en este tema, debido en gran medida a la proliferación de estas plantas en la vegetación natural y urbana (Whittaker 1984; Salas-Araiza et al. 2011; Myartseva et al. 2014, 2017; Lázaro-González et al. 2017, Chávez-Salcedo et al. 2018). Dentro de las hormigas, especies de los géneros Camponotus y Pseudomyrmex se han registrado usando nectarios extraflorales en Phoradendron tomentosum (DC.) Engelm. ex. Gray, 1849 (Whittaker 1984); Liometopum apiculatum Mayr, 1870 se reporta en trofobiosis con un hemíptero del género Saissetia que se encontraba sobre Phoradendron minutifolium Urb, 1897 (Velasco-Corona et al. 2007); Atta mexicana (Smith, 1858) se ha detectado defoliando plantas de Psittacanthus calyculatus G. Don., 1934 (Salas-Araiza et al. 2011), y las especies Camponotus crassus Mayr, 1862 C. melanoticus Emery, 1894, C. rufipes (Fabricius, 1775) у Cephalotes pusillus (Klug, 1824) se han encontrado anidado dentro del haustorio de Psittacanthus robustus Mart., 1830, y atendiendo, en la misma planta, agregaciones del hemíptero Eurystethus microlobatus Ruckes, 1966 para obtener ambrosía (Guerra et al. 2011).

En México se han registrado 927 especies, 93 géneros y once subfamilias de hormigas (Vásquez-Bolaños 2015), particularmente para el estado de Quintana Roo se conocen 171 especies en 49 géneros y nueve subfamilias (Lachaud y Pérez-Lachaud 2013). Algunos de los estudios realizados en Quintana Roo se han enfocado en hormigas arborícolas que anidan en plantas epífitas, principalmente Bromeliaceae y Orchidaceae, así como en ramas vivas y muertas del arbolado (Dejean et al. 1995; Durou et al. 2002; Suárez Vázquez 2005; Dejean et al. 2018). Este trabajo tiene como objetivo conocer la riqueza de hormigas arborícolas asociadas a plantas hemiparásitas del género Psittacanthus en el estado de Quintana Roo, para entender el papel que pueden tener las hormigas en estas plantas y las interacciones que establecen con las mismas.

\section{MATERIAL Y MÉTODOS}

Las colectas de hormigas se realizaron en una selva mediana subpereniifolia en el ejido de Felipe Carrillo Puer-

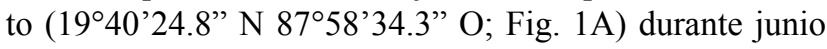

(dos colectas) y noviembre (una colecta) del año 2017. Las hormigas se colectaron en ejemplares de muérdago durante las podas realizadas por los integrantes de las Brigadas Comunitarias de Sanidad Forestal del Estado de Quintana Roo (Fig. 1B), a árboles atacados por plantas hemiparásitas (Psittacanthus mayans Standl. \& Steyerm., 1944 y $P$. rhynchanthus (Benth.) Kuijt, 1987). Las ramas afectadas se encontraban a alturas entre 7 y $18 \mathrm{~m}$, y fueron retiradas con una motosierra extensible, una vez en el suelo, se revisaron para detectar la presencia de hormigas dentro o en las ramas de planta hemiparásita o en la parte leñosa del haustorio (Fig. 1C), antes de ser trozadas. Las hormigas fueron colectadas con pinzas y aspirador entomológico según su tamaño, de las hormigas más abundantes se colectaron entre 10 y 23 ejemplares, mientras que de aquellas que eran fácilmente reconocibles como $\mathrm{D}$. bispinosus y $\mathrm{Ca}$. sericeiventris, se colectaron de 1 a 8 ejemplares, para registrar su presencia. Algunos ejemplares de cada especie fueron montados en alfileres entomológicos para su identificación, utilizando las claves especializadas para cada género (De Andrade y Baroni-Urbani 1999; Ortega-De Santiago y Vázquez-Bolaños 2012; Ward 2017; Mackay y Mackay 2019). Los ejemplares montados y en alcohol se encuentran depositados en el Laboratorio de Ecología de Artrópodos en Ambientes Extremos en la UMDI Juriquilla, UNAM.

\section{RESULTADOS Y DISCUSIÓN}

No se tomaron los datos de los árboles ni plantas hemiparásitas revisadas, ya que el objetivo de las salidas era la poda de árboles infestados en sí, y se aprovecharon las mismas para colectar las hormigas. De un total de doce árboles revisado, sólo en siete árboles pertenecientes a tres especies fueron colectadas seis especies de hormigas, el número de ejemplares colectados se presentan en el cuadro 1.

Las especies Dolichoderus bispinosus (Olivier, 1792), D. diversus Emery, 1894, Pseudomyrmex kuenckeli (Emery, 1890) y P. simplex (Smith, 1877) se colectaron en Psittacanthus mayanus infectando Lysiloma latisiliquum (L.) Benth., 1875. Mientras que Camponotus sericeiventris (Guérin-Méneville, 183) se colectó en Psittacanthus rhyntacanthus sobre Coccoloba spicata Lundell, 1939; Cephalotes biguttatus (Emery, 1890) y D. diversus también fueron colectadas en P. rhyntacanthus creciendo en Lonchocarpus rugosus Benth., 1860. Es la primera vez que estas especies son reportadas asociadas a plantas de la familia Loranthaceae. Camponotus sericeiventris fue observado anidando dentro de la rama que sostenía la planta parásita, sin embargo, no fue posible corroborar su anidación dentro de la misma (Fig. 2A). Cephalotes biguttatus se encontró anidando dentro de la estructura leñosa de la planta parásita (Fig. 2B). Dolichoderus bispinosus establece sus nidos entre las ramas de la planta parásita, en estos nidos en ocasiones viven asociadas abejas del género Trigona (Fig. 2C); en el caso de $D$. diversus se observaron galerías que iban de la planta parásita hacia el árbol soporte (Fig. 2D), sin embargo, no fue posible corroborar si anidaban dentro del haustorio. 


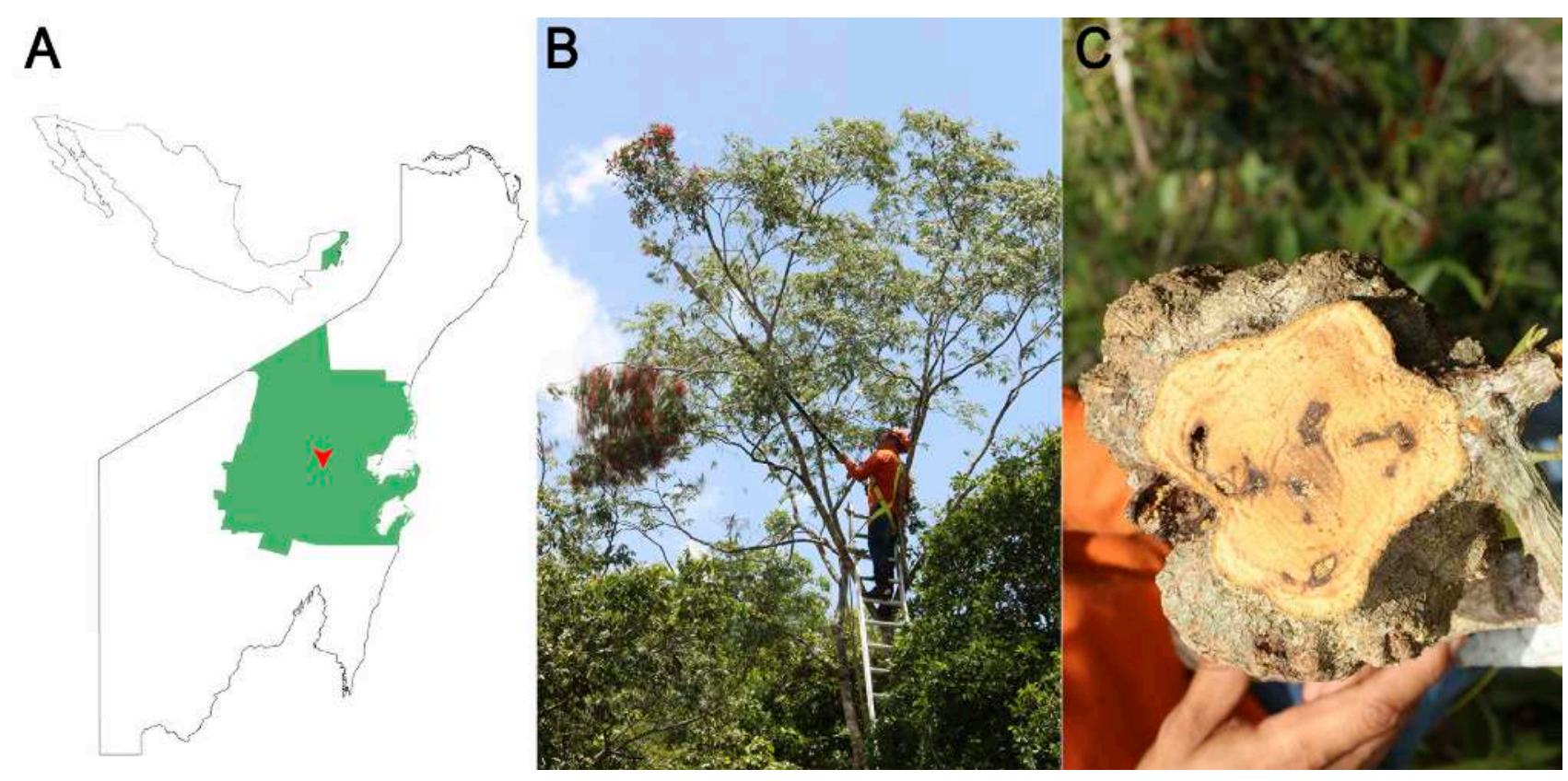

Figura 1.A sitio de estudio. 1.B Poda de plantas hemiparásitas. 1.C. Haustorio engrosado.

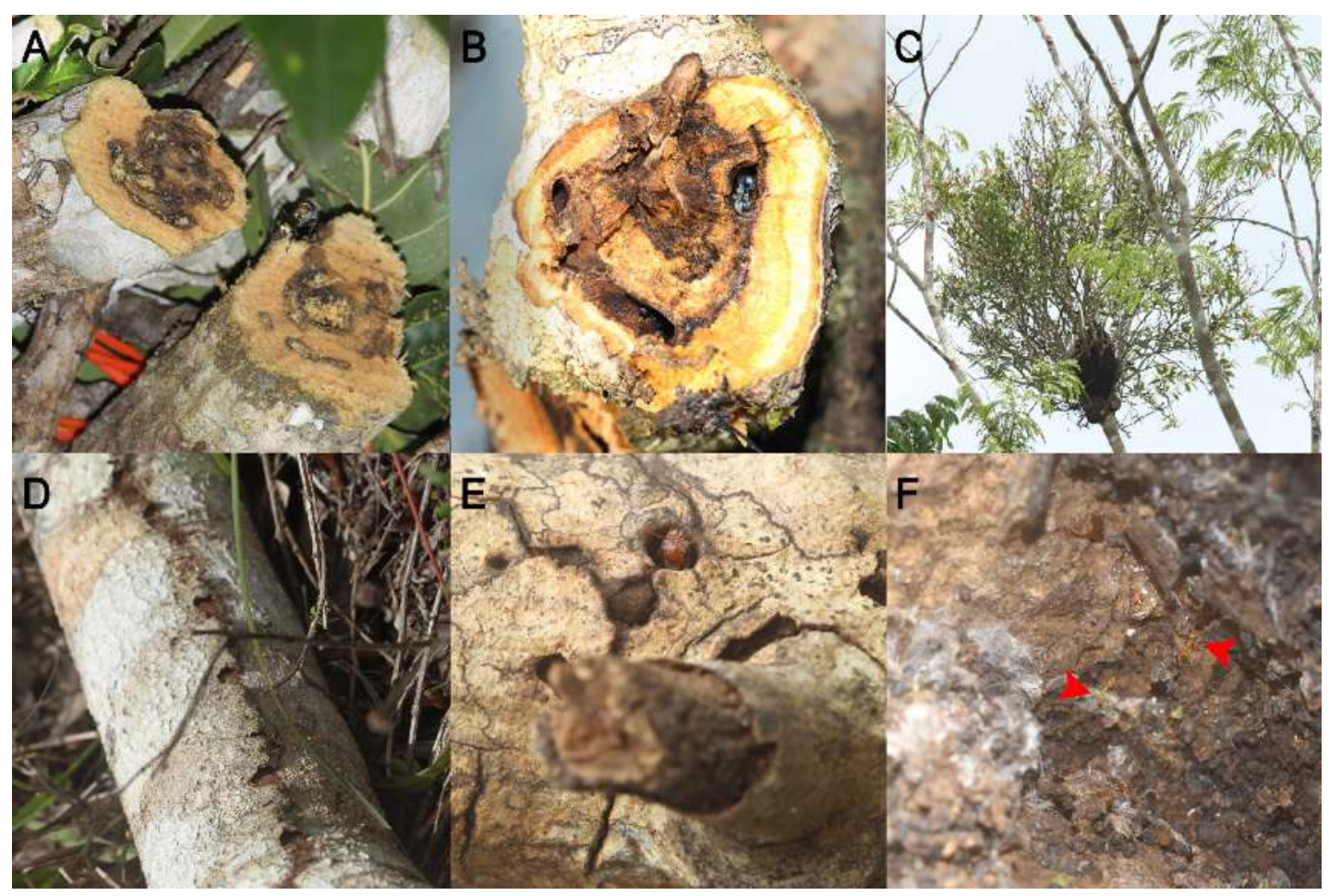

Figura 2. Especies colectadas en el presente estudio. A. Galerías de Camponotus sericeiventris. B. Cephalotes biguttatus. C. Nido de Dolichoderus bispinosus. D. Galería de D. diversus. E. Pseudomyrmex kuenckeli. F. P. simplex.

Pseudomyrmex kuenckeli fue observada anidando en orificios dentro del haustorio (Fig. 2E), mientras que P. simplex se encontró caminando entre las hojas de la planta parásita (Fig. 2F), de esta especie solo fue colectado un individuo. En selva baja subperennifolia las plantas epífitas han sido reportadas como un importante microhábitat para varias especies de hormigas (Dejean et al. 1995), mientras que en selva mediana subperennifolia se ha observado una menor ocupación y menos especies de hormigas asociadas a plantas epífitas (Dejean y Olmsted 1997). En este tipo de vegetación las plantas parásitas y hemiparásitas puedan proporcionar un refugio importante para hormigas arborícolas, durante las podas se observó una gran abundancia de plantas parásitas infestando varias especies de árboles. En época 
Cuadro 1. Especies de hormigas asociadas a plantas hemiparásitas del género Psittacanthus en Felipe Carrillo Puerto, Quintana Roo, México. () = Abundancias. I = Imagos. $\mathrm{P}=$ Pupas

\begin{tabular}{lcc}
\hline $\begin{array}{l}\text { Hormiga / Planta hemipará- } \\
\text { sita }\end{array}$ & P. mayanus & $\begin{array}{l}\text { P. rhynta- } \\
\text { canthus }\end{array}$ \\
\hline $\begin{array}{l}\text { Dolichoderinae } \\
\text { Dolichoderus bispinosus }\end{array}$ & $\mathrm{X}(\mathrm{I}: 8)$ & \\
$\begin{array}{l}\text { D. diversus } \\
\text { Formicinae }\end{array}$ & $\mathrm{X}(\mathrm{I}: 3)$ & $\mathrm{X}(\mathrm{I}: 13)$ \\
$\quad \begin{array}{l}\text { Camponotus sericeiventris } \\
\text { Pseudomyrmecinae }\end{array}$ & & $\mathrm{X}(\mathrm{I}=5)$ \\
$\quad \begin{array}{l}\text { Pseudomyrmex kuenckeli } \\
\quad \text { P. simplex }\end{array}$ & $\mathrm{X}(\mathrm{I}=10)$ & \\
Myrmicinae & $\mathrm{X}(\mathrm{I}=1)$ & \\
$\quad$ Cephalotes biguttatus & & $\mathrm{X}(\mathrm{I}=23$. \\
& & $\mathrm{P}=15)$ \\
\hline
\end{tabular}

de secas algunas especies de árboles hospederos tiran todo su follaje, mientras que las plantas parásitas mantienen la mayor parte de sus hojas, lo que probablemente mantenga un microclima más estable para artrópodos entre sus ramas. Si bien se conoce la especificidad de ciertas especies de plantas parásitas a determinados árboles hospederos (Ornelas 2019), debido a la falta de datos en la zona de estudio, no podemos saber si existe alguna preferencia de hormigas por alguna especie de Psittacanthus o un árbol hospedero. Por ejemplo, en el caso de $P$. rhyntacanthus se ha registrado como principal hospedero a Bursera simaruba Sarg., Gard. \& Forest, 1890 (Ornelas 2019), no obstante, no se tienen registros para los dos hospederos en los que fue encontrado en este estudio (Lonchocarpus rugosus y Coccoloba spicata), y las hormigas colectadas no fueron las mismas en cada caso, si bien no se realizaron colectas exhaustivas. Para el caso de Psittacanthus mayanus, se tienen registros de un mayor número de hospederos incluyendo especies del género Lysiloma, como se encontró en este estudio, así como de los géneros Lonchocarpus, Pithecellobium e Inga (Ornelas 2019). No obstante, en estudios realizados en Granada, España, se ha encontrado que las plantas hemiparásitas constituyen un ambiente particular, con una fauna asociada característica para cada especie de planta parásita, independiente de la especie de árbol que parasiten (Lázaro-González et al. 2017). Esto pone de manifiesto la necesidad de realizar más estudios para establecer si estos patrones también se presentan en la vegetación de nuestro país.

Las hormigas reportadas son especies bien representadas en el estrato arborícola, en Quintana Roo, se han reportado anidando en bromelias, así como en ramas huecas en manglar (Dejean et al. 1995; Durou et al. 2002; Suárez-Vázquez 2005) por lo que creemos que su presencia en la planta parásita no se debe a una relación estrecha. No podemos descartar que en otras especies o tipos de vegetación pudiera haber preferencia por alguna planta parásita o hospederos, por ejemplo, en selva baja perennifolia, observamos dos especies de hormigas de los géneros Camponotus y Crematogaster, diferentes de las reportadas en este estudio, anidando dentro de un mismo haustorio en Psittacanthus mayanus (Ibarra-Garibay datos no publicados).

También cabe señalar el potencial que pueden tener estas plantas como reservorios de biodiversidad. Es reconocido el papel de las epífitas en este sentido, por ejemplo, en Aechmea bracteata (Sw.) Griseb. ocupadas por Neoponera villosa (Fabricius 1804) se ha observado una gran diversidad de especies mirmecófilas, presentando interacciones interespecíficas entre varios niveles tróficos (Lachaud et al. 2017; Rocha et al. 2020). En el caso de las hemiparásitas estudiadas, además de hormigas encontramos dos hemípteros inmaduros, uno era morfológicamente parecido a Cephalotes, mientras que el otro posiblemente se desarrolla sobre la planta parásita, si bien no se observó una relación de trofobiosis como ha sido reportado entre hemípteros y hormigas en otras plantas parásitas (Blüthgen et al. 2000; Velasco-Corona et al. 2007; Guerra et al. 2011). Durante las podas en dos ocasiones se observaron hormigueros de Dolichoderus bispinosus asociados a una especie de Trigo$n a$ no identificada. Previamente se han registrado abejas sin aguijón anidando junto a hormigas, sin embargo, en muchos casos se considera ocasional y accidental (Sakagami et al. 1989). Existe un reporte de D. bispinosus asociación con Paratrigona opaca (Cockerell, 1917) en Colombia, en un nido probablemente hecho por Azteca sp y que posteriormente ocupó D. bispinosus (Bequaert 1943).

Los estudios enfocados en la artropodofauna en plantas parásitas son escasos, pero han demostrado la gran riqueza de ordenes asociados (Whittaker 1984; Chávez-Salcedo et al. 2018). Considerando que muchas de estas plantas se encuentran en el dosel de las selvas, que es el estrato menos conocidos y que actualmente las plantas parásitas son consideradas un problema a nivel nacional (Alvarado y Saavedra 2017), es necesario dirigir los esfuerzos para comprender su artropodofauna asociada y su relación con la planta parásita, así como el papel que pueden tener las hormigas en estas plantas y las interacciones que establecen con las mismas. No hay que olvidar que estas plantas también proveen recurso y refugio para una gran diversidad de animales (Tun Tun y Martínez-Ambriz, 2018; Ornelas 2019), así como una gran riqueza y abundancia de artrópodos (Chávez-Salcedo et al. 2018).

En años recientes, diversos estudios han puesto en evidencia la necesidad de reconsidera el papel del parasitismo en los ecosistemas, pues su papel en las redes tróficas puede ser muy relevante y modificar las interacciones en las mismas redes (Lafferety et al. 2008; Preston y Johnson 2010; Frainer et al. 2018), por lo que también el estudio de la fauna que se asocia a las plantas epífitas resulta de sumo interés. Los resultados obtenidos muestran el gran potencial que puede tener el estudio de la fauna asociada a muérda- 
gos, en particular las hormigas, para comprender mejor la biodiversidad, pues pueden resultar un ambiente propicio para una fauna particular.

\section{AGRADECIMIENTOS}

Este trabajo se dedica con profunda admiración y respeto al Dr. Alfonso Neri García Aldrete, en reconocimiento de su gran trayectoria y agradecimiento por sus contribuciones a la entomología en México, en particular en el estudio de los psocópteros. Se agradece a las integrantes de las Brigadas Comunitarias de Sanidad Forestal del estado de Quintana Roo por el apoyo en el trabajo de campo. La Dra. Alicia Callejas Chavero (ENCB-IPN) y un revisor anónimo, proporcionaron valiosas sugerencias para mejorar el trabajo.

\section{LITERATURA CITADA}

Alvarado, D. y L. de L. Saavedra. 2017. La investigación sobre plantas parásitas en México. (pp. 7-14). In: Alvarado, D. y L. de L. Saavedra (Eds.). Memorias del Foro Nacional: Las Plantas Parásitas en México. Colegio de Postgraduados, Campus Montecillo. Texcoco, estado de México.

Bequaert, J.C. 1943. Stingless bees nesting in association with ants (Hymenoptera). Bulletin of the Brooklyn Entomological Society (new series), 36:141.

Blüthgen, N., M. Verhaagh, W. Goitía, K. Jaffé, W. Morawetz y W. Barthlott. 2000. How plants shape the ant community in the Amazonian rainforest canopy: the key role of extrafloral nectaries and homopteran honeydew. Oecologia, 125(2): 229-240.

Chávez-Salcedo, L.F., M.E. Queijeiro-Bolaños, V. López-Gómez, Z. Cano-Santana, B.E. Mejía-Recamier y A. Mojica-Guzmán. 2018. Contrasting arthropod communities associated with dwarf mistletoes Arceuthobium globosum and A. vaginatum and their host Pinus hartwegii. Journal of Forestry Research, 29: 1351-1364.

CONAFOR, 2006. Fondo Sectorial para la Investigación, el Desarrollo y la Innovación Tecnológica Forestal. Demanda 1.11 Ecología y manejo de plantas parásitas y epífitas en ecosistemas forestales del semidesierto mexicano. En: http:/www.conacyt.mx/fondos/FondosSectoriales/CONAFOR/Convocatoria $\% 20 \mathrm{CONA-FORCO-}$ NACYT\%20200601/CONAFOR_Demandas_2006-01. pdf. Fecha de consulta: 11-01-2020.

De Andrade, M.L. y C. Baroni-Urbani. 1999. Diversity and adptation in the ant genus Cephalotes, past and present. Stuttgarter Beiträge zur Naturkunde, Serie B, 271: 1-889.

Dejean, A. y Olmsted, I. 1997. Ecological studies on Aechmea bracteata (Swartz) (Bromeliaceae). Journal of Natural History, 31:1313-1334.

Dejean, A., I Olmsted y R.R. Snelling. 1995. Tree-epiphyte-ant relationships in the low inundated forest of Sian Ka'an Biosphere Reserve, Quintana Roo, Mexico. Biotropica, 27 (1):57-70.
Dejean, A., A. Compin, M. Leponce, F. Azémar, C. Bonhomme, S. Talaga, L. Pelozuelo, Y. Hénaut y B. Corbara. 2018. Ants impact the composition of the aquatic macroinvertebrate communities of a myrmecophytic tank bromeliad. Comptes Rendus Biologies, 341: 200207.

Durou, S., A. Dejean, I. Olmsted y R.R. Snelling. 2002. Ant diversity in coastal zones of Quintana Roo, Mexico, with special reference to army ants. Sociobiology, 40 (2): 385-402.

Frainer, A., B.G. McKie, P.A. Amundsen, R. Knudsen y K.D. Lafferty. 2018. Parasitism and the biodiversity-functioning relationship. Trends in Ecology \& Evolution, 33: 260-268.

Geils, B.W., J. Cibrian y B. Moody. 2002. Mistletoes of North American Conifers. Gen. Tech. Rep. RMSRSGRT-98. Ogden, UT: U.S. Department of Agriculture, Forest Service, Rocky Mountain Research Station.

Guerra, T.J., F. Camarota, F.S. Casto, C.F. Schwertner y J. Grazia. 2011. Trophobiosis between ants and Eurystethus microlobatus Ruckes 1966 (Hemiptera: Heteroptera: Pentatomidae) a cryptic, gregarious and subsocial stinkbug. Journal of Natural History, 45: 1101-1117.

Lachaud, J.P. y G. Pérez-Lachaud. 2013. Revisión preliminar de las hormigas de Campeche y Quintana Roo, México, con base en la colección de Arthropoda del Colegio de la Frontera Sur. (pp. 23-32). En: Vásquez-Bolaños, M., G. Castaño-Meneses, A. Cisneros-Caballero, G.A. Quiroz-Rocha y J.L. Navarrete-Heredia (Eds.). Formicidae de México. Orgánica Editores, México.

Lachaud, J.-P., F. Rocha, y G. Pérez-Lachaud. 2017. Parasitic organisms associated with the ponerine ant $\mathrm{NeO}$ ponera villosa nesting in tank bromeliads in the Yucatan Peninsula, Mexico. 7th Central European Workshop of Myrmecology. Cracovia, Polonia.

Lafferty, K.D., S. Allesina, M. Arim, C.J. Briggs, G. De Leo, A.P. Dobson, J.A. Dunne, P.T.J. Johnson, A.M. Kuris, D.J. Marcogliese, N.D. Martinez, J. Memmott, P.A. Marquet, J.P. McLaughlin, E.A. Mordecai. M. Pascual, R. Poulin y D.W. Thieltges. 2018. Parasites in food webs: the ultimate missing links. Ecology Letters, 11: 533-546.

Lázaro-González, A., J.A. Hódar y R. Zamora. 2017. Do the arthropod communities on a parasitic planta and its hosts differ? European Journal of Entomology, 114: 215-221.

Mackay, W.P. y E.E. Mackay. 2019. Género Camponotus. Capítulo 26. (pp. 743-789). En: Fernández, F., R.J. Guerrero y T. Delsinne (Eds.). Hormigas de Colombia. Universidad Nacional de Colombia, Bogotá, Colombia.

Martínez-Ambriz, E. 2017. Flora del Valle de Tehuacán-Cuicatlán. Fascículo 140. Loranthacear Juss. Instituto de Biología, UNAM, México, D. F.

Myartseva, S.N., E. Ruiz-Cancino y J.M. Coronodo-Blanco. 2014. Dos especies nuevas del género Coccophagus Westwoo, 1833 (Hymenoptera: Aphelinidae) del estado 
de Tamaulipas, México. Acta Zoológica Mexicana, 30 (1): 135-143.

Myartseva, S.N., E. Ruiz-Cancino y J.M. Coronodo-Blanco. 2017. Primer registro de Diversinervus elegans Silvestri (Hymenoptera: Encyrtidae) en el estado de Tamaulipas, México, y otros parasitoides de la escama Parasaissetia nigra (Nietner) (Hemiptera: Coccidae). Entomología Mexicana, 16 (2): 777-780.

Ornelas, J.F. 2019. Los Muérdagos Pssitacanthus en México: ecología, evolución, manejo y conservación. Biodiversitas, 146:12-16.

Ortega-De Santiago, J.L. y M. Vásquez-Bolaños. 2012. Especie nueva de Dolichoderus (Hymenoptera: Formicidae) de Puerto Vallarta, Jalisco y nuevos registros para México. Revista Mexicana de Biodiversidad, 83 (4): 1004-1008.

Press, M.C. y G.K. Phoenix. 2005. Impacts of parasitic plants on natural communities. New Phytologist, 166 (3): 737-751.

Press, M.C., J.D. Scholes y J.R. Watling. 1999. Parasitic plants: physiological and ecological interactions with their hosts. (pp. 175-197). In: Press, M.C., J.D. Scholes and M.G. Barker (Eds.). Physiological Plant Ecology. Blackwell Science, Oxford, UK.

Preston, D. y P. Johnson. 2010. Ecological consequences of parasitism. Nature Education Knowledge, 1 (8): 39-47.

Rocha, F. H., J.-P. Lachaud y G. Pérez-Lachaud. 2020. Myrmecophilous organisms associated with colonies of the ponerine ant Neoponera villosa (Hymenoptera: Formicidae) nesting in Aechmea bracteata bromeliads: a biodiversity hotspot. Myrmecological News, 30: 73-92.

Sakagami, S., T. Inoue, S. Yamane, y S. Salmah. 1989. Nests of the Myrmecophilous Stingless Bee, Trigona moorei: How do Bees Initiate Their Nest Within an Arboreal Ant Nest? Biotropica, 21(3): 265-274.

Salas-Araiza, M.D., R.W. Jones y R. Ramírez-Malagón.

Recibido: 17 febrero 2020

Aceptado: 14 mayo 2020
2011. Herbivores of Psittacantus calyculatus Don. (Loranthaceae) in Mexico: a parasitic plant of mesquite. Southwestern Entomologist, 36 (1): 107-110.

Suárez Vázquez, H.D. 2005. Forrajeo y anidamiento de hormigas (Hymenoptera: Formicidae) en epífitas mirmecófilas de Río Hondo, México. Tesis profesional. Licenciado en Biología, Departamento de Ingenieria Quimica y Bioquimica, Instituto Tecnológico de Chetumal, Quintana Roo, México.

Tun-Garrido, J. 2010. Plantas parasitas y hemiparásitas. http://www.cicy.mx/Documentos/CICY/Sitios/Biodiversidad/pdfs/Cap4/21\%20Plantas\%20parasitas.pdf. Fecha de consulta: 20-02-2020.

Tun Tun, C.M y E. Martínez-Ambriz. 2018. Psittacanthus mayanus y la familia Loranthaceae en Yucatán. Desde el Herbario CICY 10: 29-33. https:// www.cicy.mx/Documentos/CICY/Desde_Herbario/2018/2018-02-15-CTun-EMartinez-Psittacanthus-mayanus.pdf Fecha de consulta: 07-05-2020.

Vásquez-Bolaños, M. 2015. Taxonomía Formicidae (Hymenoptera) para México. Métodos en Ecología y Sistemática, 10 (1): 1-53.

Velasco-Corona, C., M. del C. Corona-Vargas y R. Peña-Martínez. 2007. Liometopum apiculatum (Formicidae: Dolichoderinae) y su relación trofobiótica con Hemiptera Sternorrhyncha en Tlaxco, Tlaxcala, México. Acta Zoológica Mexicana (n.s), 23 (2): 31-42.

Villaseñor, J.L. 2016. Catálogo de las plantas vasculares nativas de México. Revista Mexicana de Biodiversidad, 87 (3): 559-902.

Ward, P.S. 2017. A review of the Pseudomyrmex ferrugineus and Pseudomyrmes goeldii species groups: acacia-ants and relatives (Hymenoptera: Formicidae). Zootaxa, 4227: 524-542.

Whittaker, P.L. 1984. The insect fauna of mistletoe (Phoradendron tomentosum, Loranthaceae) in Southern Texas. Southwestern Naturalist, 29 (4): 435-444. 